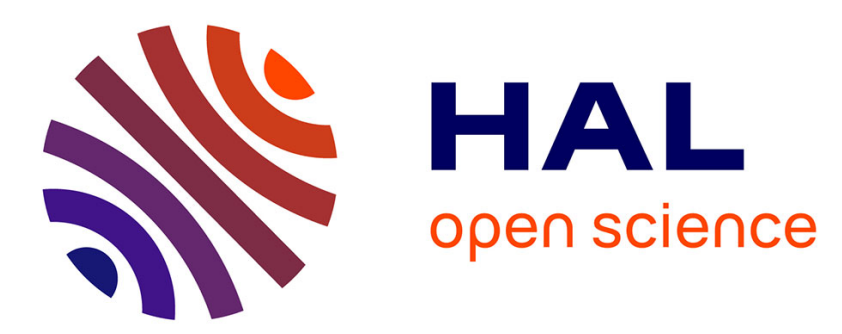

\title{
AxTract: microstructure-driven tractography based on the ensemble average propagator
}

\author{
Gabriel Girard, Rutger H.J. Fick, Maxime Descoteaux, Rachid Deriche,
} Demian Wassermann

\section{- To cite this version:}

Gabriel Girard, Rutger H.J. Fick, Maxime Descoteaux, Rachid Deriche, Demian Wassermann. AxTract: microstructure-driven tractography based on the ensemble average propagator. Information Processing in Medical Imaging, Jun 2015, Isle of Skye, United Kingdom. 10.1007/978-3-319-199924_53. hal-01152267

\section{HAL Id: hal-01152267 \\ https://inria.hal.science/hal-01152267}

Submitted on 18 May 2015

HAL is a multi-disciplinary open access archive for the deposit and dissemination of scientific research documents, whether they are published or not. The documents may come from teaching and research institutions in France or abroad, or from public or private research centers.
L'archive ouverte pluridisciplinaire HAL, est destinée au dépôt et à la diffusion de documents scientifiques de niveau recherche, publiés ou non, émanant des établissements d'enseignement et de recherche français ou étrangers, des laboratoires publics ou privés. 


\title{
AxTract: microstructure-driven tractography based on the ensemble average propagator
}

\author{
Gabriel Girard $^{1,2}$, Rutger Fick ${ }^{1}$, Maxime Descoteaux ${ }^{2}$, Rachid Deriche ${ }^{1}$ and \\ Demian Wassermann ${ }^{1}$ \\ ${ }^{1}$ Athena Project-Team, INRIA Sophia Antipolis - Méditerranée, France and \\ ${ }^{2}$ Sherbrooke Connectivity Imaging Lab (SCIL), Computer Science Department, \\ Université de Sherbrooke, Canada
}

\begin{abstract}
We propose a novel method to simultaneously trace brain white matter (WM) fascicles and estimate WM microstructure characteristics. Recent advancements in diffusion-weighted imaging (DWI) allow multi-shell acquisitions with b-values of up to $10,000 \mathrm{~s} / \mathrm{mm}^{2}$ in human subjects, enabling the measurement of the ensemble average propagator (EAP) at distances as short as $10 \mu \mathrm{m}$. Coupled with continuous models of the full 3D DWI signal and the EAP such as Mean Apparent Propagator (MAP) MRI, these acquisition schemes provide unparalleled means to probe the WM tissue in vivo. Presently, there are two complementary limitations in tractography and microstructure measurement techniques. Tractography techniques are based on models of the DWI signal geometry without taking specific hypotheses of the WM structure. This hinders the tracing of fascicles through certain WM areas with complex organization such as branching, crossing, merging, and bottlenecks that are indistinguishable using the orientation-only part of the DWI signal. Microstructure measuring techniques, such as AxCaliber, require the direction of the axons within the probed tissue before the acquisition as well as the tissue to be highly organized. Our contributions are twofold. First, we extend the theoretical DWI models proposed by Callaghan et al. to characterize the distribution of axonal calibers within the probed tissue taking advantage of the MAP-MRI model. Second, we develop a simultaneous tractography and axonal caliber distribution algorithm based on the hypothesis that axonal caliber distribution varies smoothly along a WM fascicle. To validate our model we test it on in-silico phantoms and on the HCP dataset.
\end{abstract}

\section{Introduction}

Diffusion-weighted (DW) magnetic resonance imaging (MRI) has empowered the analysis of the brain's white matter (WM) anatomy and its relationship with function and pathologies in recent years. DWI has provided great tools to advance the study of neuroscience and neuropathology. However, the relationship between the measures obtained from the DWI signal and their underlying biological process is still unclear. In this work, we build upon current advances in DWI acquisition and signal modelling to develop a new technology to trace WM 
fascicles while simultaneously characterizing the distribution of axonal calibers (i.e. diameters) within the fascicle. In doing this, we expect to provide better tools to characterize the WM. Examples of these possible applications could be pathology-oriented, for instance through the detection of axonal swelling and quantification of the connectivity between two cortical areas; or neuroscience oriented, as axonal caliber has been shown to be closely related to the efficiency of electrical signal propagation within the axons [12].

Despite recent advances in axonal caliber quantification from DWI, to the best of our knowledge, none of them has been used to improve tractography at the moment of the tract tracing process. Two approaches to combine microstructure information and tractography have been proposed [9, 14]. To solve complex WM areas (e.g. branching, crossing, merging, and bottlenecks), these approaches reject tracts from a full brain tractography based on microstructure information. This is sensitive to the choice of the tractography algorithm used, since this requires all plausible configurations of tracts inside the complex region. Our novel tractography algorithm, AxTract, addresses the complex configuration problem differently. AxTract incorporates the axonal caliber estimation in the tractography algorithm and uses it during the tracing process. This produces tracts with embedded microstructure information and enables the possibility of solving the tracing through WM areas using axonal calibers information.

In developing AxTract to be useful for reasonably long DWI acquisitions, we base our model solely on the ensemble average propagator (EAP) [11] at a fixed gradient separation time. This relaxes the requirements of axonal caliber estimation techniques such as AxCaliber [3] and ActiveAx [1] which focus on the signal attenuation and need a sampling over different gradient separation times. Our approach has the main advantage of simultaneously modelling, through the Fourier slice theorem, all measurements on the perpendicular plane to the cylinder population. To prove the soundness of our model, we developed the first contribution of this work: a generalized return-to-axis probability (RTAP) measure [11] showing that even at gradient separation times used in current clinical protocols, axonal caliber can be quantified. This enabled our second contribution: the AxTract algorithm which estimates axonal caliber during the tractography process using it as a prior for the traced tract.

\section{Theory}

The DWI signal within a voxel measures diffusion of water particles within different compartments, such as axons and astrocytes. It is possible to characterize the diffusion process as the displacement probability density of water particles within these compartments, the ensemble average propagator (EAP) [11]:

$$
\bar{P}\left(\boldsymbol{r}-\boldsymbol{r}^{\prime} ; \Delta\right)=\sum_{c \in C} \rho_{c}\left(\boldsymbol{r}^{\prime}\right) \int_{\mathbb{R}^{3}} P_{0}\left(\boldsymbol{r}^{\prime} ; c\right) P_{c}\left(\boldsymbol{r} ; \boldsymbol{r}^{\prime}, \Delta\right) d \boldsymbol{r}^{\prime}
$$

where $\boldsymbol{r}^{\prime}, \boldsymbol{r}$ are the particle's start and end positions; $\Delta$ the diffusion time; $C$ is the set of compartments; $\rho_{c}\left(\boldsymbol{r}^{\prime}\right)$ is the probability of $\boldsymbol{r}^{\prime}$ being inside compartment 
$c ; P_{0}$ the probability of the initial position in $c$; and $P_{c}$ the compartment-specific propagator. The EAP is related to the attenuation of the DWI signal by the Fourier transform:

$$
E(\boldsymbol{q} ; \Delta)=\mathcal{F}_{\boldsymbol{r}-\boldsymbol{r}^{\prime}}\left\{\bar{P}\left(\boldsymbol{r}-\boldsymbol{r}^{\prime} ; \Delta\right)\right\}(\boldsymbol{q})
$$

Within the study of the human brain WM, the axons present a specific interest. Within a voxel, axons can be modelled as cylindrical segments, for which specific formulations of $P$ and $E$ exist [6]. In compartments where diffusion takes place within a set of cylinders oriented along direction $\boldsymbol{r}_{\|}$with negligible tortuosity and permeability, the displacement $\boldsymbol{r}-\boldsymbol{r}^{\prime}$ is decomposable in the parallel and perpendicular directions to the cylinder [4]. We write this as $\boldsymbol{r}-\boldsymbol{r}^{\prime}=$ $\left(\boldsymbol{r}_{\|}-\boldsymbol{r}_{\|}^{\prime}\right)+\left(\boldsymbol{r}_{\perp}-\boldsymbol{r}_{\perp}^{\prime}\right)$ leading to separable formulations of $P$ and $E$ [4]:

$$
\begin{aligned}
\bar{P}\left(\boldsymbol{r}-\boldsymbol{r}^{\prime} ; \Delta\right) & =\bar{P}\left(\boldsymbol{r}_{\|}-\boldsymbol{r}_{\|}^{\prime} ; \Delta\right) \bar{P}\left(\boldsymbol{r}_{\perp}-\boldsymbol{r}_{\perp}^{\prime} ; \Delta\right) \\
& =\int_{\mathbb{R}} P_{0}\left(\boldsymbol{r}_{\|}^{\prime}\right) P_{c_{\|}}\left(\boldsymbol{r}_{\|}-\boldsymbol{r}_{\|}^{\prime} ; \Delta\right) d \boldsymbol{r}_{\|}^{\prime} \int_{\mathbb{R}^{2}} P_{0}\left(\boldsymbol{r}_{\perp}^{\prime}\right) P_{c_{\perp}}\left(\boldsymbol{r}_{\perp}-\boldsymbol{r}_{\perp}^{\prime} ; \Delta\right) d \boldsymbol{r}_{\perp}^{\prime} \\
E(\boldsymbol{q} ; \Delta) & =E\left(\boldsymbol{q}_{\|} ; \Delta\right) E\left(\boldsymbol{q}_{\perp} ; \Delta\right) .
\end{aligned}
$$

This decomposition enables the use of theoretical models for $P_{c}$ [6]. If the propagator $P_{c}$ is measured at a cylinder of cross-sectional area $A$ and it's filled with water with diffusion coefficient $D$, we derive the following expressions [6]:

$$
\begin{aligned}
& P_{c y l_{\|}}\left(\boldsymbol{r}_{\|} ; \boldsymbol{r}_{\|}^{\prime}, \Delta\right)=\frac{e^{-\frac{\left\|\boldsymbol{r}_{\|}-\boldsymbol{r}_{\|}^{\prime}\right\|^{2}}{4 D \Delta}}}{\sqrt{2 \pi D \Delta}} \\
& P_{c y l_{\perp}}\left(\boldsymbol{r}_{\perp} ; \boldsymbol{r}_{\perp}^{\prime}, \Delta, A\right)=\sum_{n k} 2^{1_{n=0}}\left(e^{-\frac{\pi \gamma_{n k}^{2} D \Delta}{A}} \frac{\gamma_{n k}^{2}}{\mathrm{~J}_{n}\left(\gamma_{n k}^{2}\right)\left(\gamma_{n k}^{2}-n^{2}\right) A}\right. \\
&\left.\mathrm{J}_{\mathrm{n}}\left(\frac{\sqrt{\pi} \gamma_{n k}\left\|\boldsymbol{r}_{\perp}\right\|}{\sqrt{A}}\right) \mathrm{J}_{\mathrm{n}}\left(\frac{\sqrt{\pi} \gamma_{n k}\left\|\boldsymbol{r}_{\perp}^{\prime}\right\|}{\sqrt{A}}\right) \cos (n \theta) \cos \left(n \theta^{\prime}\right)\right)
\end{aligned}
$$

where $1_{n=0}$ is the indicator function for $n=0, \theta$ and $\theta^{\prime}$ are the respective angles of $\boldsymbol{r}_{\perp}$ and $\boldsymbol{r}_{\perp}^{\prime}$ when expressed in polar coordinates, $\mathrm{J}_{n}$ is the $\mathrm{n}$-th cylindrical Bessel function and $\gamma_{k n}$ the $k$-th the root of its derivative: $\mathrm{J}_{n}^{\prime}\left(\gamma_{k m}\right)=0$.

Having a specific model for cylindrical compartments, i.e. axons, we can derive the theory to estimate the axonal cross-sectional area. Different techniques to capitalize the theoretical model in Equation (4) for cylinder compartments and measure axonal radii have been proposed. The main exponents of these are AxCaliber [3]; ActiveAx [1]; and the Return-to-Axis-Probability (RTAP) [11]. However, the applicability of these techniques is limited, even in current stateof-the-art whole human brain acquisitions such as the HCP project: AxCaliber relies on a relatively dense sampling along the $q$ and $\Delta$ dimensions; ActiveAx estimates a single-parameter which experimentally correlates with the mean caliber without an explicit formal relationship to it; and RTAP which needs very 
large diffusion times $(\Delta)$ for the perpendicular EAP to become [11]

$$
\bar{P}_{c y l_{\perp}}\left(\boldsymbol{r}_{\perp} ; A, \Delta\right) \stackrel{\Delta \rightarrow \infty}{\longrightarrow} \frac{4 \cos ^{-1}\left(\frac{\left\|\boldsymbol{r}_{\perp}\right\| \sqrt{\pi}}{2 \sqrt{A}}\right)-\frac{\left\|\boldsymbol{r}_{\perp}\right\| \sqrt{\pi}}{2 \sqrt{A}} \sqrt{4-\frac{\left\|\boldsymbol{r}_{\perp}\right\|^{2} \pi}{A}}}{2 \pi A}
$$

and converge to the reciprocal of the cross-sectional area of the axonal population

$$
\operatorname{RTAP}(\Delta)=\int_{\mathbb{R}^{2}} E_{\perp}\left(\boldsymbol{q}_{\perp} ; \Delta\right) d \boldsymbol{q}_{\perp}=\bar{P}_{\perp}(\mathbf{0}, \Delta) \stackrel{\Delta \rightarrow \infty}{\longrightarrow} A^{-1} .
$$

The first contribution of our work is to prove that, even at small $\Delta$ values, the propagator along the cylinder has a specific relationship with the distribution of cross-sectional areas in a cylinder population. We base our model on the EAP as opposed to the AxCaliber and RTAP approaches which focus on the signal attenuation. This has the main advantage of simultaneously modelling, through the Fourier slice theorem, all measurements on the perpendicular plane to the cylinder population. We start our model in the style of AxCaliber and "infinite $\Delta$ " RTAP, we attach a density to the cross-sectional area of a cylinder population. Our density is based on three hypotheses given by Özarslan et al. [11]. First, each particular cylinder's contribution to the overall signal is proportional to the ratio of water particles in it, which is in direct relationship with the cylinder's cross-sectional area. Second, the cylinder population is Gamma-distributed [3]. This leads to specific EAP formulation, Equation (1), for $N$ cylinders:

$$
\bar{P}\left(\boldsymbol{r}-\boldsymbol{r}^{\prime} ; \Delta, \alpha, \beta, N\right)=\sum_{i=1}^{N} \frac{A_{i}}{\sum_{j}^{N} A_{j}} \bar{P}_{c y l}\left(\boldsymbol{r}-\boldsymbol{r}^{\prime} ; \Delta, A\right), A_{i} \sim \Gamma(\alpha, \beta),
$$

where each $A_{i}$ is an independent and identically distributed random variable with Gamma distribution, of shape $\alpha$ and rate $\beta$, of the cross-sectional area. Finally, our third hypothesis assumes that the population is large enough to be approximated by an infinite number of cylinders. Combining Equations (1) and (5)

$$
\begin{aligned}
& \bar{P}\left(\boldsymbol{r}-\boldsymbol{r}^{\prime} ; \Delta, \alpha, \beta\right)=\lim _{N \rightarrow \infty} \bar{P}\left(\boldsymbol{r}-\boldsymbol{r}^{\prime} ; \Delta, \alpha, \beta, N\right)= \\
& \quad \int_{0}^{\infty} \frac{A f(A ; \alpha, \beta)}{\alpha \beta^{-1}} \bar{P}_{c y l}\left(\boldsymbol{r}-\boldsymbol{r}^{\prime} ; \Delta, A\right) d A
\end{aligned}
$$

where the integral over $A$ takes in account all possible cross-sectional areas; $f(A ; \alpha, \beta)$ is the probability density function of a Gamma distribution with shape $\alpha$ and rate $\beta$; and $\alpha \beta^{-1}$ is the average cross-sectional area under the distribution $\Gamma(\alpha, \beta)$. By using the separability of the EAP (see Equation (3)) and assuming a uniform probability of finding a water particle within the cylinder population, we marginalize Equation (6) for the return-to-axis probability, i.e. $\boldsymbol{r}_{\perp}^{\prime}=\boldsymbol{r}_{\perp}$,

$$
\bar{P}_{\perp}(\mathbf{0} ; \Delta, \alpha, \beta)=\int_{0}^{\infty} \frac{A f(A ; \alpha, \beta)}{\alpha \beta^{-1}} \int_{\mathbb{R}^{2}} P_{0}(\boldsymbol{r} ; A) P_{c y l_{\perp}}(\boldsymbol{r} ; \boldsymbol{r}, \Delta, A) d \boldsymbol{r} d A
$$


where $P_{0}(\boldsymbol{r} ; A)$ is the uniform distribution of $\boldsymbol{r}$ within the disc of surface $A$.

Then, replacing Equation (4) into Equation (7), we reach our first result

$$
\begin{aligned}
\bar{P}_{\perp}(\mathbf{0} ; \Delta, \alpha, \beta) & =\frac{(D \Delta \pi)^{\frac{\alpha-1}{2}}}{\beta^{\frac{-1-\alpha}{2}} \Gamma(\alpha)} \sum_{n k} 2^{1_{n=0}} \gamma_{n k}^{\alpha} \mathrm{K}_{\alpha-1}\left(2 \gamma_{n k} \sqrt{D \Delta \pi \beta}\right) \mathrm{R}_{n}\left(\gamma_{n k}\right) \\
\mathrm{R}_{\mathrm{n}}(\gamma) & \triangleq \frac{\gamma\left(\mathrm{J}_{n}^{2}(\gamma)+\mathrm{J}_{n+1}^{2}(\gamma)\right)-2 n \mathrm{~J}_{n}(\gamma) \mathrm{J}_{n+1}(\gamma)}{\mathrm{J}_{\mathrm{n}}^{2}(\gamma)\left(\gamma^{2}-\mathrm{n}^{2}\right)}
\end{aligned}
$$

where $\mathrm{K}$ is the modified Bessel function. Finally, the calculating $P_{\perp}(\mathbf{0} ; \Delta, \alpha, \beta)$ from the 3D signal combining Equations (3), (4) and (8) is the same as RTAP

$$
\int_{0}^{\infty} \bar{P}\left(\boldsymbol{r}_{\|} r+\boldsymbol{r}_{\perp} 0\right) d r=\int_{0}^{\infty} \bar{P}_{\|}(r ; \Delta) d r \bar{P}_{\perp}(\mathbf{0} ; \Delta, \alpha, \beta)=\frac{1}{2} \bar{P}_{\perp}(\mathbf{0} ; \Delta, \alpha, \beta)
$$

This characterization of $P_{\perp}$ in the case of an axonal population has two main advantages: first, it doesn't depend on a very large $\Delta$ for its relationship with the axonal radii to be true; second, as a corollary of the relationship between the EAP $P_{\perp}$ and the attenuation $E_{\perp}$, Equation (8) aggregates information from all measurements perpendicular to the cylinder population and, by using a full 3D model with analytic Fourier transform such as Mean Apparent Propagator (MAP) MRI [11], takes advantage of the full extent of q-space measurements in the acquisition protocol.

\section{Methods}

\subsection{AxTract: Microstructure-driven Tractography}

Model Fitting The main purpose of our novel tractography algorithm, AxTract, is to simultaneously trace WM fascicle and estimate their axonal caliber. The main hypothesis driving AxTract is that the average caliber of the axons composing a tract varies slowly along its pathway.

To formulate our algorithm we start from the classical equation driving streamline tractography [5]:

$$
\frac{d \boldsymbol{t}(s)}{d s}=\boldsymbol{d}(s), \quad \boldsymbol{t}(0)=\boldsymbol{t}_{0}
$$

where the curve $\boldsymbol{r}(s)$ is the streamline tracing the WM fascicle that traverses $\boldsymbol{t}_{0}$, and $\boldsymbol{d}(s)$ is the tangent vector to $\boldsymbol{t}(s)$, and taken to be the eigenvector corresponding to the maximal eigenvalue of the diffusion tensor (DT) at $\boldsymbol{t}(s)$. More generally, using the DTI EAP model, $\boldsymbol{d}(s)$ is equivalent to the direction of maximal diffusion probability

$$
\boldsymbol{d}(s)=\arg \max _{\hat{\boldsymbol{r}} \in \mathbb{R}^{3},\|\hat{\boldsymbol{r}}\|=1} \mathrm{ODF}(\hat{\boldsymbol{r}} ; s)
$$

where ODF is the orientation distribution function [17]

$$
\mathrm{ODF}(\hat{\boldsymbol{r}})=\int_{0}^{\infty} \bar{P}(\hat{\boldsymbol{r}} r) \rho(r) d r, \quad \hat{\boldsymbol{r}} \in \mathbb{R}^{3},\|\hat{\boldsymbol{r}}\|=1
$$


with $\rho(r)$ a function of the area element of the sphere, such as $\rho(r)=r^{2}$ [16].

Algorithms based on Equation (10) rely on the hypothesis that fiber tracts are locally tangent to direction of maximal diffusion probability. Specifically, the DT model cannot express complex geometries such as tract crossings and kissings. Hence, several algorithms have been proposed to extend this algorithm and be able to trace through these geometries [e.g. 15, 16, 17]. These algorithms rely on the same hypothesis of that the direction of maximum probability is enough to trace these tracts and add, in one way or another, a new hypothesis of preservation of the previous tracking direction.

With AxTract, we aim to preserve not only direction but average axonal caliber, adding a biologically-driven hypothesis and enabling to traverse complex structures with more confidence on the results. At each point along the tract, we fit a model of the EAP based on the theoretical models presented in Section 2. We develop a multi-compartment fitting model to separate the EAP data corresponding to a particular population from other compartments. Hence, we assign to each ODF peak an EAP formulation for a cylindrical population and we add to the whole ensemble an isotropic tensor representing the combination of extracellular water and spherical compartments such as astrocytes. The formulation of our tract-point model

$$
\begin{aligned}
& \bar{P}_{f i t}\left(\boldsymbol{r} ; D, f_{1}, \ldots, f_{N}, A_{1}, \ldots, A_{N}, d_{1}, \ldots, d_{N}\right)= \\
& \quad \sum_{i}^{N} f_{i} \bar{P}_{c y l}\left(\boldsymbol{r} ; A_{i}, d_{i}\right)+\left(1-\sum_{i}^{N} f_{i}\right) \bar{P}_{e c}(\boldsymbol{r} ; D), \quad \sum_{i}^{N} f_{i} \leq 1, f_{i} \in[0,1]
\end{aligned}
$$

where $N$ is the number of non-collinear tracts crossing that point, namely of ODF peaks; $A_{i}$ the average calibers; $d_{i}$ the tract orientation; $\bar{P}_{c y l}$ the propagator of a cylindrical ensemble, where for implementation speed, we assume a large diffusion time. $\bar{P}_{e c}(\boldsymbol{r}, D)$ is the extra-cylindrical compartment propagator:

$$
\bar{P}_{e c}(\boldsymbol{r}, D)=\frac{\exp \left(-\frac{1}{4 D \tau} \boldsymbol{r}^{T} \boldsymbol{r}\right)}{\sqrt{2 \pi D \tau}^{3}}, \quad D \in \mathbb{R}_{>0}
$$

and $f$ are the mixing factors.

Then, for a given an EAP $\bar{P}(\boldsymbol{r})$, we fit our model in Equation (13) by minimizing the squared loss function with combined global-local optimisers

$\underset{D, f_{1}, \ldots, f_{N}, A_{1}, \ldots, A_{N}}{\operatorname{argmin}}=\int_{\mathbb{R}^{3}}\left[\bar{P}(\boldsymbol{r})-\bar{P}_{f i t}\left(\boldsymbol{r} ; D, f_{1}, \ldots, f_{N}, A_{1}, \ldots, A_{N}, d_{1}, \ldots, d_{N}\right)\right]^{2} d \boldsymbol{r}$

To fit Equation (15) and to extract the ODF peaks, we use a continuous representation of $\bar{P}$ which is analytically estimated from the DWI signal attenuation, MAP-MRI [11]. This provides us with the means to obtain the ODF peaks and optimize our objective function over a dense sampling on $\boldsymbol{r}$.

Tractography Streamlines propagate in a WM volume and stop when a position outside the volume is reached [15]. The tracking process propagates a seed 
from the initial position (placed within the volume) following diffusion properties. To obtain these properties, the signal at the tracking position is obtained with trilinear interpolation of the DWIs. Then, MAP-MRI is used to represent the signal locally [11], from which the ODF peaks are extracted. Using the peak directions and the signal representation from MAP-MRI, AxTract estimates each peak's caliber (see Equation (15)). The streamline propagation follows the peak with the estimated axonal caliber closest to the median caliber of the current streamline. The estimated streamline caliber is given by median caliber over previous tracking directions for a fixed maximum distance of $5 \mathrm{~mm}$. Peaks forming an angle greater than $\theta=75^{\circ}$ with the previous tracking direction are discarded from the selection to enforce smoothness in streamline reconstruction [15]. The tracking stops if no peaks are available. The initial tracking direction is set to the direction associated with the maximum value of the ODF locally. Once the tracking stops, it is re-initiated in the opposite initial direction to form the complete streamline. Additionally, we fixed the tracking discrete step size to $0.5 \mathrm{~mm}$.

\section{Dataset}

\subsection{Human Dataset}

We used the Human Connectome Project (HCP) MGH adult diffusion dataset (subject mgh1010) [13]. The diffusion acquisition scheme consists of 552 volumes with b-values ranging from 1000 to $10,000 \mathrm{~s} / \mathrm{mm}^{2}(\delta=12.9 \mathrm{~ms}, \Delta=21.8 \mathrm{~ms}$ ). The data were acquired using at $1.5 \mathrm{~mm}$ isotropic voxel size using a Spin-echo EPI sequence (TR/TE $8800 / 57 \mathrm{~ms}$ ).

\subsection{Synthetic Dataset}

We used Phantomas [7] to generate in silico data using the acquisition scheme of the HCP dataset. The DWI signal is simulated in each voxel based on the Numerical Fiber Generator [8]. The simulated signal is obtained using a hindered and restricted diffusion model [2], and adding Rician noise. Synthetic data with signal-to-noise ratio (SNR) of 20 and 100 are used in this study.

\section{$5 \quad$ Results}

Figure 1 shows the error in caliber estimation using Equation (15), varying the axon population caliber. Figure $1(\mathrm{a}, \mathrm{b})$ shows the caliber estimation in a single axon population per voxel. Figure $1(\mathrm{c}, \mathrm{d})$ shows caliber etimation of two axon populations crossing at $90^{\circ}$ in a voxel $(\mathrm{SNR}=100,20)$, with a constant axon caliber of $12 \mu \mathrm{m}$ for the second axon population. Figure 2 shows estimated calibers in the corpus callosum (CC) on in vivo data. The algorithm is able to recover various calibers that are consistent locally, with lower caliber in the genu and in the splenium than in the body of the CC (see Figure 2 (b)). 


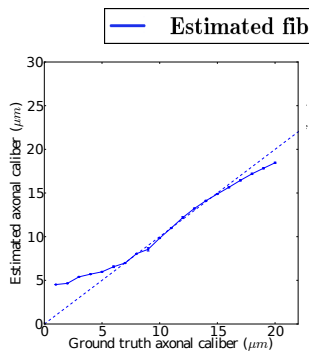

(a) Single $(\mathrm{SNR}=100)$

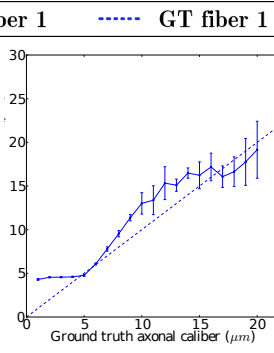

(b) Single $(\mathrm{SNR}=20)$

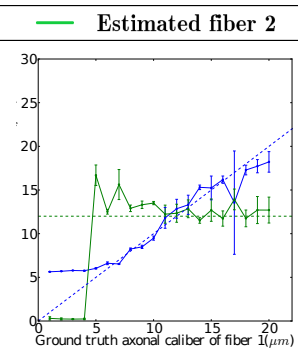

(c) Crossing $(\mathrm{SNR}=100)$

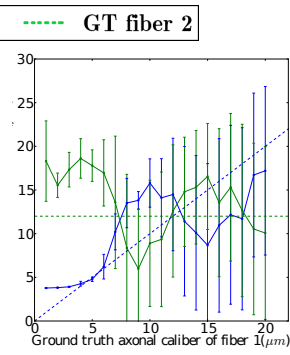

(d) Crossing $(\mathrm{SNR}=20)$

Fig. 1. Error on synthetic fiber calibers estimation using Equation (15). (a, b) Single fiber caliber estimation. The ground truth caliber is shown by the dashed blue line. (b, d) $90^{\circ}$ crossing fibers caliber estimation. The second fiber has a constant caliber of $12 \mu m$ shown by the dashed green line.

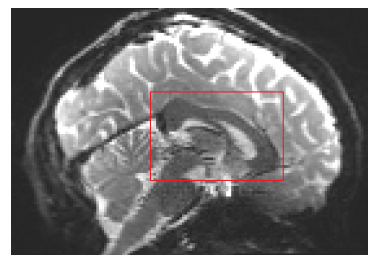

(a) B0 image

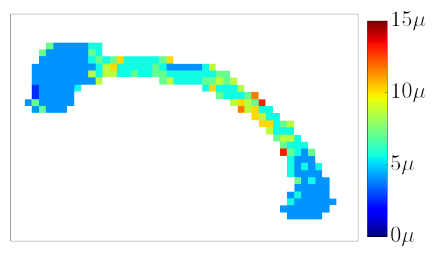

(b) Peak calibers

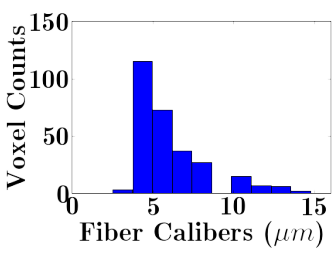

(c) Calibers histogram

Fig. 2. Axonal caliber estimation in the CC using HCP data. Caliber was estimated in the maximum direction of the ODF. Calibers are lower in the genu and in the splenium than in the body of the CC.

We further test Equation (15) in kissing configuration. Figure 3 (a-c) shows the synthetic kissing dataset. The right fiber $(4 \mu \mathrm{m})$ is predominant on the ODFs image (see Figure 3 (a)). This can also be observed in the EAP at low radius (Figure 3 (b)). At higher radii (Figure 3 (c)), both fibers are visible in crossing regions. However, when both fiber crosses at less than $20^{\circ}$, only one peak appears and it is biased in the direction of the fiber with the smallest axonal caliber. ODFs peaks are shown in Figure 3 (d). Figure $3(\mathrm{e}, \mathrm{f})$ shows in each voxel the peak with the highest and lowest caliber value. This can be observed quantitatively in Figure 4 on the histogram of peaks caliber and on the caliber map, for both $\mathrm{SNR}=100$ and $\mathrm{SNR}=20$.

Figure 5 shows a comparison of deterministic ODF tractography and AxTract on a synthetic kissing configuration. All streamlines connecting valid fascicle extremities are shown in blue (left fiber fascicle) or green (right fiber fascicle) and other streamlines (invalid or incomplete) are shown in red. The deterministic tractography is not able reconstruct the fascicle on the left side. Streamlines are deviated in the right fascicle which has higher diffusion properties. Single peak voxel tends to be biased in the direction the fiber with the lowest caliber, as 


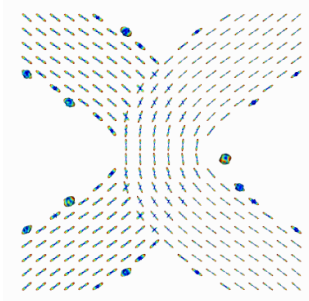

(a) ODFs

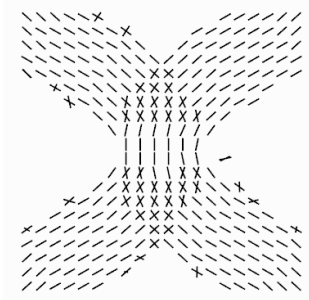

(d) Peaks

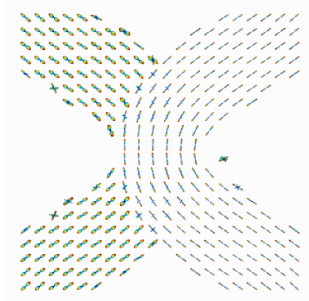

(b) $\operatorname{EAP}_{(r=0.009 \mu m)}$

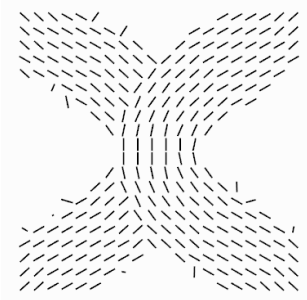

(e) Min. caliber

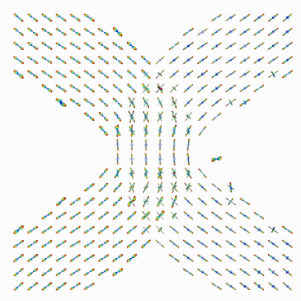

(c) $\operatorname{EAP}_{(r=0.021 \mu \mathrm{m})}$

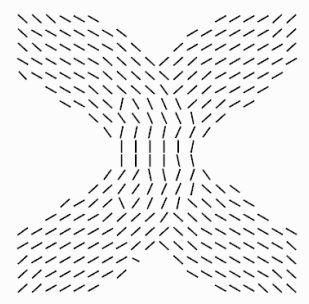

(f) Max. caliber

Fig. 3. Synthetic kissing configuration dataset at $\mathrm{SNR}=20$. The fiber on the left has a caliber of $12 \mu \mathrm{m}$ and the fiber on the right has a caliber of $4 \mu \mathrm{m}$. (a) ODFS, (b, c) EAP value at fixed radii $r,(\mathrm{~d})$ peaks extracted from ODFs, (e, f) peaks with the minimum, respectively the maximum, caliber in each voxel.

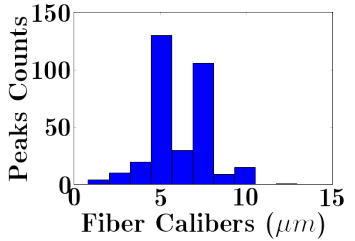

(a) Calibers hist. (SNR=100)

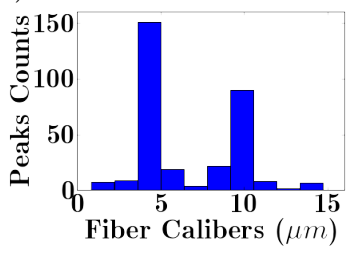

(c) Calibers hist. ( $\mathrm{SNR}=20)$
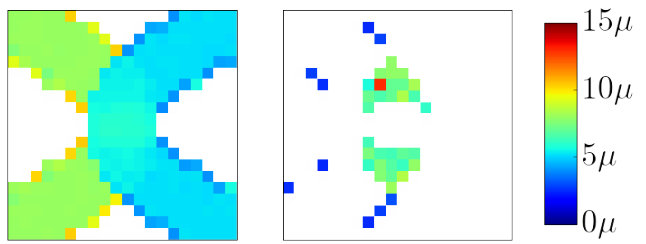

(b) Peak calibers $(\mathrm{SNR}=100)$
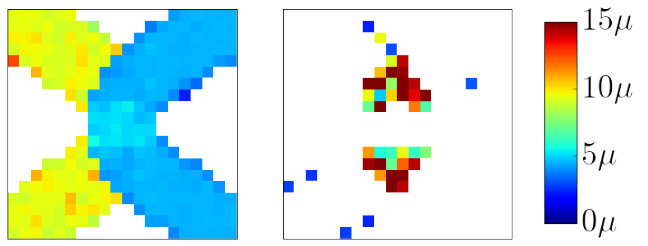

(d) Peak calibers $(\mathrm{SNR}=20)$

Fig. 4. Peaks caliber estimation using Equation (15). The fiber on the left has a caliber of $12 \mu \mathrm{m}$ and the fiber on the right has a caliber of $4 \mu \mathrm{m}$. (a, c) Histograms of estimated calibers for all peaks, (b, d) spacial maps of peaks with lowest caliber estimated on the left and the highest caliber on the right.

shown in Figure 3 (d). AxTract, using microstructure information, successfully reconstructed both WM fascicles (see Figure $5(\mathrm{~b}, \mathrm{~d})$ ). 


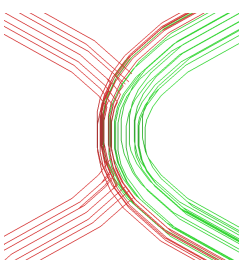

(a) Det. $(\mathrm{SNR}=100)$

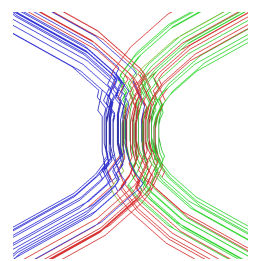

(b) AxTract (SNR=100)

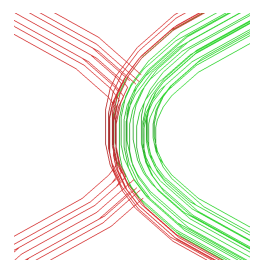

(c) Det. $(\mathrm{SNR}=20)$

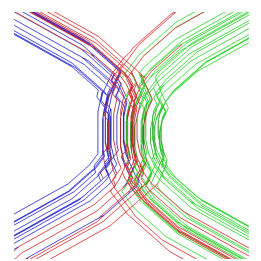

(d) AxTract (SNR=20)

Fig. 5. Comparison of deterministic ODF tractography and AxTract on synthetic kissing configuration (left fascicle caliber $=12 \mu \mathrm{m}$, right fascicle caliber $=4 \mu \mathrm{m}$ ). All streamlines connecting valid fascicle extremities are shown in blue (left fiber) and green (right fiber), other streamlines are shown in red. The deterministic tractography is not able to reconstruct the fascicle on the left side. Streamlines deviate in the right fascicle with lower caliber. Although not perfect, AxTract is able to recover valid streamlines from both fascicles and reduced the number of invalid connections.

\section{Discussion and Conclusion}

In this paper, we introduced a novel algorithm to simultaneously perform tractography and axonal caliber estimation. In doing this, our algorithm is able to use the axonal caliber as a prior to trace fascicles through complex WM areas such as kissings.

Simplified parametric formula for the EAP in a cylindrical population: The first contribution of our work is to establish the grounds for axonal caliber measurement on DWI acquisitions which holds when the diffusion time $\Delta$ is short. We based our analysis on the theoretical models for diffusivity within cylinders provided by [6] and derived a series-based expression of the EAP at limited $\Delta$ in terms of the axonal caliber. Our resulting formula Equation (8) shows that, even at limited $\Delta$, RTAP is an estimator of axonal caliber. Moreover, through the Fourier slice theorem, the EAP along the axis parallel to the cylinder population aggregates information of the signal attenuation on whole plane perpendicular to the cylinders, providing a simplified mean to use all information available from a multi-shell acquisition for caliber estimation.

Average Axonal caliber is measurable through the EAP at limited $\Delta$ : Having grounds for using RTAP to measure axonal caliber at limited $\Delta$, we proceeded to test its sensitivity in different scenarios. Figure 1 (a) showed, in synthetic experiments, that RTAP clearly distinguishes various axonal calibers in single axon population cases. This is further supported by Figure 2 where various calibers are recovered in the human corpus callosum. As show by McNab et al. [10], the recovered calibers are generally higher in the body of the CC than in the genu and the splenium of the CC. However, no axonal calibers difference was observed between the genu and the splenium of the CC in this single subject. Further investigation is required to quantify the difference between AxCaliber axonal caliber estimation and AxTract. Nevertheless, Figure 2 shows that axon populations with various caliber can be identified in vivo, which can be used within the choice of the propagation direction of the tractography. 
AxTract effectively solves kissings using axonal caliber as a prior: In fiber crossing regions, AxTract distinguishes axonal caliber population, specially at low noise level (see Figures 1 and 4). Even though the estimation is not as accurate as in single population cases, it showed improvements in the streamline reconstruction, both at $\mathrm{SNR}=20$ and $\mathrm{SNR}=100$ (Figure 5). The median caliber estimated locally seems to be robust to misestimation of the caliber at some of the tracking steps. We can observe in Figure 5 that the tractography needs in some cases to follow peaks with higher local deviations to keep following the fascicle with the closest caliber. Always following peaks with the lowest local deviation, as with deterministic peak tractography, leads to error in the kissing configuration reconstruction. A smaller tracking step size would help following the fascicle tangent direction and reduce the maximum deviation angle $\theta$ parameter, thus producing a smoother WM pathway reconstruction. AxTract allows the streamline propagation to follow pathways otherwise not explored by the deterministic tractography. Those results are preliminary, but expose a potential use of the microstructural information in tractography to properly reconstruct complex WM architectures such as kissing and branching configurations. Future work on full brain WM reconstruction is planned. The main limitation of the current implementation is the computational requirements.

To conclude, in this work we presented AxTract, a novel algorithm that uses simultaneous axonal caliber estimation and tractography to resolve WM fascicle tracking through areas of complex WM configurations. In order to develop this algorithm, we provided mathematical grounds for the feasibility of caliber estimation and evidence for both in silico and human data. Finally, we tested our tractography algorithm which was able to tract through a fiber kissing using a priori information of the traced fascicle which was previously not possible.

Acknowledgments. Human brain data were provided by the Human Connectome Project (HCP; Principal Investigators: Bruce Rosen, M.D., Ph.D., Arthur W. Toga, Ph.D., Van J. Weeden, MD). HCP funding was provided by the National Institute of Dental and Craniofacial Research, the National Institute of Mental Health, and the National Institute of Neurological Disorders and Stroke.

\section{References}

[1] Alexander, D.C., Hubbard, P.L., Hall, M.G., Moore, E.A., Ptito, M., Parker, G.J., Dyrby, T.B.: Orientationally invariant indices of axon diameter and density from diffusion MRI. NImg 42, 1374-1389 (2010)

[2] Assaf, Y., Basser, P.J.: Composite hindered and restricted model of diffusion (CHARMED) MR imaging of the human brain. NImg 27(1), 48-58 (2005)

[3] Assaf, Y., Blumenfeld-Katzir, T., Yovel, Y., Basser, P.J.: AxCaliber: a method for measuring axon diameter distribution from diffusion MRI. MRM 59, 1347-1354 (2008)

[4] Assaf, Y., Freidlin, R.Z., Rohde, G.K., Basser, P.J.: New modeling and experimental framework to characterize hindered and restricted water diffusion in brain white matter. MRM 52, 965-978 (2004) 
[5] Basser, P.J., Pajevic, S., Pierpaoli, C., Duda, J., Aldroubi, A.: In vivo fiber tractography using DT-MRI data. MRM 44, 625-632 (2000)

[6] Callaghan, P.: Pulsed-Gradient Spin-Echo NMR for Planar, Cylindrical, and Spherical Pores under Conditions of Wall Relaxation. Journal of Magnetic Resonance, Series A 13, 53-59 (1995)

[7] Caruyer, E., Daducci, A., Descoteaux, M., Houde, J.c., Thiran, J.p., Verma, R.: Phantomas: a flexible software library to simulate diffusion MR phantoms. In: International Symposium on Magnetic Resonance in Medicine (2014)

[8] Close, T.G., Tournier, J.D., Calamante, F., Johnston, L.a., Mareels, I., Connelly, A.: A software tool to generate simulated white matter structures for the assessment of fibre-tracking algorithms. NImg 47(4), 1288-1300 (2009)

[9] Daducci, A., Dal Palu, A., Alia, L., Thiran, J.P.: COMMIT: Convex Optimization Modeling for Micro-structure Informed Tractography. IEEE Transactions on Medical Imaging 34, 246-257 (2014)

[10] McNab, J.a., Edlow, B.L., Witzel, T., Huang, S.Y., Bhat, H., Heberlein, K., Feiweier, T., Liu, K., Keil, B., Cohen-Adad, J., Tisdall, M.D., Folkerth, R.D., Kinney, H.C., Wald, L.L.: The Human Connectome Project and beyond: initial applications of $300 \mathrm{mT} / \mathrm{m}$ gradients. NImg 80, 234-245 (2013)

[11] Özarslan, E., Koay, C.G., Shepherd, T.M., Komlosh, M.E., İrfanoğlu, M.O., Pierpaoli, C., Basser, P.J.: Mean apparent propagator (MAP) MRI: A novel diffusion imaging method for mapping tissue microstructure. NImg 78, 16$32(2013)$

[12] Ritchie, J.M.: On the relation between fibre diameter and conduction velocity in myelinated nerve fibres. Proceedings of the Royal Society of London. Series B, Containing papers of a Biological character. Royal Society (Great Britain) 217(1206), 29-35 (1982)

[13] Setsompop, K., Kimmlingen, R., Eberlein, E., Witzel, T., Cohen-Adad, J., McNab, J., Keil, B., Tisdall, M., Hoecht, P., Dietz, P., Cauley, S., Tountcheva, V., Matschl, V., Lenz, V., Heberlein, K., Potthast, A., Thein, H., Horn, J.V., Toga, A., Schmitt, F., Al., E.: Pushing the limits of in vivo diffusion MRI for the Human Connectome Project. NImg 80, 220-233 (2013)

[14] Sherbondy, A.J., Rowe, M.C., Alexander, D.C.: MicroTrack: An Algorithm for Concurrent Projectome and Microstructure Estimation. In: International Conference on Medical Image Computing and Computer-Assisted Intervention. pp. 183-190 (2010)

[15] Tournier, J.D., Calamante, F., Connelly, A.: MRtrix: Diffusion tractography in crossing fiber regions. International Journal of Imaging Systems and Technology 22(1), 53-66 (2012)

[16] Tristán-Vega, A., Westin, C.F., Aja-Fernández, S.: Estimation of fiber orientation probability density functions in high angular resolution diffusion imaging. NImg 47, 638-650 (2009)

[17] Tuch, D.: Q-ball imaging. MRM 52, 1358-1372 (2004) 